\title{
Biomedical

\section{Differentiation of human fat mesenchymal stem cells using electromagnetic waves}

\author{
Nariman Gharari ${ }^{1}$ and Mahsa Laleh $^{2}$ \\ ${ }^{1}$ School of Biology, University of Tehran, Tehran, Iran \\ ${ }^{2}$ Department of Biomedical Engineering, Azad University of Science and Research Branch, Tehran, Iran
}

\begin{abstract}
In orthopedic sciences, cartilage regeneration and repairing is an underlying issue investigated by many studies in tissue engineering. Bioactive growth factors, cell implantation and biocompatible scaffolding are the new developments in this field. Keeping the proliferation and differentiation ability of cultured cells is essential in producing extracellular matrix and cartilage. Adult stem cells can be used due to their high ability to be differentiated in several cell types in tissue engineering. Till recently hematopoietic stem cells and fat tissue stem cells have been used to repair the tissues. Studies indicates that using fat tissue stems cells is preferred because of easy access to adipose tissue and maintaining the ability of proliferation and differentiation to cartilage in high passages compared to hematopoietic stem cells. Therefore, this study has been conducted to analyze the differentiation of human fat mesenchymal stem cells using electromagnetic waves. In this work simultaneous effect of platelet-rich plasma and electromagnetic waves have been studied on efficiency of differentiation of fat mesenchymal stem cells to cartilage. Mesenchymal stem cells have been extracted from human fat tissue and then, the cells have been differentiated to fat, bone and cartilage tissue cells using different treatments. Cartilage differentiation was done in 2-D form and single-layer cell in bottom of 6-bed and 3-D cells in cell falcons. Required tests to estimate efficiency of cartilage differentiation were done in two general examinations for analysis of evaluation of specific and pathologic markers such as osteogenicity, angiogenesis and inflammation parameters by measuring the amount of TNF and VEGF with ELISA technique. The results obtained from this study showed reasonable use of platelet-rich plasma (PRP) and electromagnetic waves in medicine and tissue engineering. Although PRP enables cartilage differentiation, it cannot reduce pathologic symptoms.
\end{abstract}

KEY WORDS: DIFFERENTIATION OF HUMAN FAT MESENCHYMAL STEM CELLS, ELECTROMAGNETIC WAVES, PRP

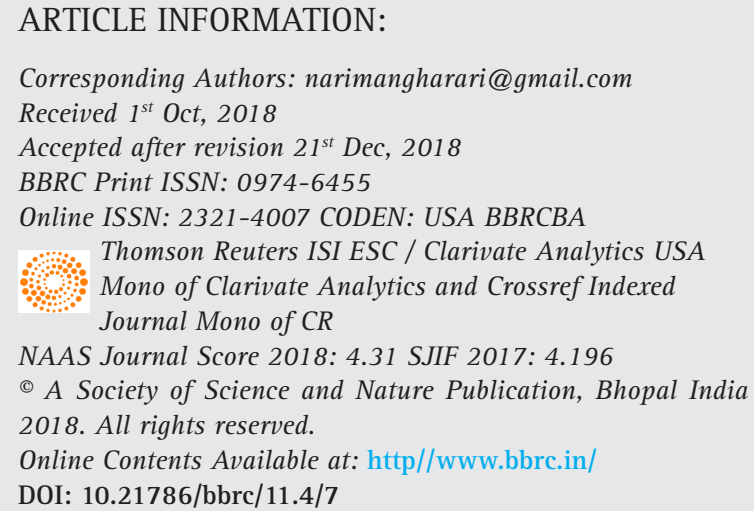




\section{INTRODUCTION}

The aim of tissue engineering is normalizing tissue performance through transferring living factors in body of patients, (Kocan et al. 2017, Bondarava et al. 2017). Continuous efforts of cell biologists, engineers, material engineers, mathematicians, geneticists and physicians should be in way of achievement and success in new tissue production, (Alonso-Goulart et al. 2017, Klar et al. 2017).

Nowadays, tissue engineering is being used as a technique for tissue or organ transplant. The lost and injured tissues and organs can be treated by engineered biological transplant (Zhai et al. 2016). Engineered products and components should have high performance and have the ability to form expected functional tissue (Zajdel et al. 2017). Mesenchymal stem cells (Skeletal Stem Cells or Bone marrow stromal stem cells) are plastic sticky non-hepatocytes cells placed in bone marrow stromal vessels (Seong et al. 2014 Ross et al. 2015 and Liu et al. 2017) and have multi-generation differentiation with self-renewal capacity (Heo et al. 2016 and Mobini et al. 2017). Deciding which type of cell should be applied to repair injured tissue is essential which can be adult brucellosis, mesenchymal stem cells or protozoal cells from hypothyroidism, pericardium or cells manipulated genetically, (Tan et al. 2017). Cartilage is non-vascular fibrous connective tissue having resistant extracellular matrix, which is stiffer than other connective tissues and includes intra-matrix chondrocytes, (Choi et al. 2018).

The first function of cartilage is protection of soft tissues and helping evolution and growth of long bones. Fat tissue stem cells are similar to bone marrow stromal cells in terms of physical form and can be appropriate replacement. These cells have common features compare with mesenchymal stem cells such as self-renewal ability, powerful cells, fibroblast appearance, ability to attach to plastic dishes for cell cultivation and ability to be changed into other mesenchymal types, (Dubois et al. 2008 and Tan et al. 2017).The differentiation of these cells from fat, bone, cartilage, skeletal muscular and cardiovascular cells has been reported in recent years by different researchers. Fat tissue stem cells, same as bone marrow stromal cells, can be converted to ectodermal types (like neural and glial cells) by the mediation of neural inducers, (Ivan et al. 2017).

Several studies have been conducted in fat tissue stem cells, especially for treatment of neural system lesions and revealed that direct injection of fat stem cells to brain ischemic models can convert these cells to quasineural cells. These cells can improve laboratory models in the place of lesion with (injured place by) secretion of neural growth factors (Gugjoo et al. 2016).In order to prepare mesenchymal cell, fat tissue is a considerable source, since it is available in large amount (liposuctioned) fat tissue or in fat tissue section and contains large amount of stem cells called fat-derived stem cells. The property has made it be considered as a good candidate for 1-step treatment of cartilage defects (Bruder et al. 1997).

In the past decade, PRP or platelet-rich plasma has gained many attentions, especially in regenerative medicine, (Liu et al. 2017). PRP can be considered as a part of blood plasma with platelet density higher than base level (Xu et al. 2017 Qian et al. 2017). Importance of PRP with appropriate density of platelet is the abundant growth factors and proteins used in tissue engineering, (Anitua, et al. 2007). In 2012, a study was done in China for regenerating cartilage, which evaluated and compared mesenchymal stem cells, extracted from bone marrow and fat tissue placed on the PRP-derived scaffolding. Findings showed that rich plasma is appropriate bioactive scaffolding with ability of secreting growth factor, bone marrow stem cells and fat mesenchymal stem cells -placed on PRP were differentiated from cartilage and efficient to repair cell-based cartilage, (Lee et al. 2012).

In a study, the effects of platelet-rich plasma on histological, biochemical and biomechanical properties of cartilage tissue engineering were studied. Cultured cells in PRP created 20\% thicker cartilage tissue compared with other cultured cells. Hence, platelet-rich plasma in culture medium can cause creation of cartilage in vitro cartilage formation with increased content of glycosaminoglycan and more mechanical compressive properties at the same time with maintaining Phenotype Features of Hyaline cartilage (Petrera, et al. 2013). In 2014, a research team from Spain studied effects of PRP on human mesenchymal stem cells, which can increase or limit clinical uses of these cells. PRP can preserve differentiated immune of mesenchymal stem cells and can apparently postpone ageing phenomenon. They also provide data on exact molecular relation and its mechanisms, (Rubio-Azpeitia et al. 2014).

In 2014, an economic method was proposed to prepare PRP. PRP-derived growth factor-BB was measured under conditions of using Anticoagulant dextrose solution A (ACD-A), along with or in absence of Prostaglandin E1 (PGE1) (as Platelet aggregation inhibitor). The new method was successful in analysis of PRP with growth factor and high $\mathrm{BB}$ under all growth conditions and high volume of PRP is obtained using ACD-A and PGE1(Fukaya et al. 2014).

In 2014, the effect of PRP on cartilage cells differentiated from rabbit fat-derived stem cells was studied in vitro. Stem nature of rabbit cells were studied with differentiation of fat, bone and cartilage types in vitro. Collagen type 2 expression and agrican expression in PRP-treated cells was increased to 10\% compared to 
control group. Hence, the findings showed that PRP of rabbit can take cartilage differentiation in mesenchymal stem cells extracted from rabbit fat (Elder et al. 2014).

Analysis of the simultaneous effect of PRP and electromagnetic waves on cartilage differentiation efficiency in fat mesenchymal cells has been studied. Mesenchymal stem cells have been extracted from human fat tissue and then, the cells were differentiated to types of fat tissues, bone and cartilage using different treatments Therefore, these experiment have been conducted to analyze differentiation of mesenchymal human fat stem cells using electromagnetic waves.

\section{MATERIALS AND METHODS}

Extraction of mesenchymal stem cells from fact tissue:To transfer fat tissue, fat pieces were placed in DMEM medium containing Penicillin and streptomycin $3 \mathrm{X}$, amphotericin $1 \mathrm{x}$, without FBS and were transferred to laboratory in ice. After each time of adding buffer to 2-3 times of tissue volume, centrifuge was taken with speed of 1200rpm for 20min and buffer solution was disposed. A thin layer of fat was formed on the solution, which had to be removed, since it could cause problems such as choking on cells due to lack of oxygen while cultivation. In this step, suspended cells were transferred to flasks 75 containing $10 \mathrm{ml}$ culture medium with FBS and were then heated in incubator.

To make cells face electromagnetic waves, after counting cells using Neubauer lam method, a part of culture medium containing 200.000 cells was removed from culture flasks and was transferred to sterile falcons. Then, $1 \mathrm{ml}$ of culture medium without differentiation factors and containing Penicillin - Streptomycin and FBS was added and maintained for 1 day. After the heating in incubator, cells were accumulated in bottom of the falcon in rounded form and were prepared to continue the examination and to expose to electromagnetic radiation. To apply electromagnetic waves on cell culture, wave winding machine was used. The winding machine was placed inside the incubator and culture falcon was placed there to be exposed to electromagnetic waves for 6 hours per day. Frequency of the machine was set in range $171-173 \mathrm{~Hz}$ and according to power about 30\%, ultimate and real time power about $50 \mathrm{~Hz}$ was obtained. Cells were exposed to such conditions in 14-day period and the culture medium was changed every 3 days. 5 falcons containing culture medium with 5\% PRP were exposed to electromagnetic waves and other 4 falcons were not. Measurement of VEGF distributed in differentiated cells was done using supernatant of cultures in days 7, 14 and 21. The phosphorylation of this factor was measured by kit based on Sandwich ELISA method.
The Sandwich ELISA method was used to measure the amount of TNF $\alpha$ propagated from differentiating cells. To this end, the supernatant collected from cell culture was examined in the days 7, 14 and 21. The final results were analyzed statistically at the confidence level of 95\% to test significance of the difference of different groups using Prism software and using one-way ANOVA and T-test. Moreover, the diagrams were drawn using Excel and SPSS software. The results obtained from analysis of cartilage differentiation gene expression were analyzed using REST software by normalization with reference beta-actin gene. Each test was replicated 3 times and the significance was considered lower than 0.05.

\section{RESULTS}

Analysis of differentiation of stem cells extracted from fat tissue to cartilage :In this study, differentiation to cartilage was analyzed using different staining methods and Immunocytochemistry test during 14 days of cell differentiation. Expression of some genes relevant to cartilage was also studied at the end of the differentiation period.

Proof of cartilage differentiation: The Immunocytochemistry method was used to analyze expression and production of collagens types 2 and 10. Collagen type 2 was expressed during cartilage differentiation and collagen type 10 was one of the bone and hypertrophy markers expressed by chondrocytes. Figure 1 has illustrated expression of these collagens in treated cells in the day 14. The blue color in the figure is DAPI staining to stain cell core and red color shows presence of studied collagens in differentiating cells.

Analysis of bone markers during differentiation of stem cells to cartilage: At the time of applying differentiation factors such as using PRP as a bioactive substance or electromagnetic radiation to stimulate cartilage differentiation in mesenchymal stem cells of fat tissue, non-specified differentiation to bone is also possible. With taking tests such as measurement of Alkaline Phosphatase Activity and Calcium sedimentation measurements, the non-specified differentiation was also examined.

Measurement of Alkaline Phosphatase Activity: To determine non-specified differentiation towards bones, measurement of alkaline phosphatase enzyme was activity was done. As it is clear in diagram 1, the amount of alkaline phosphatase in treatment PRP.W is the highest level measured equal to 2 units of optical absorption per mg protein. Then, treatment PRP a with value of 1.44 unit absorption per mg protein shows highest alkaline phosphatase activity. The lowest level of phosphatase 


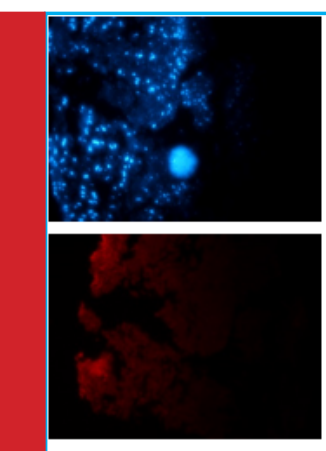

A
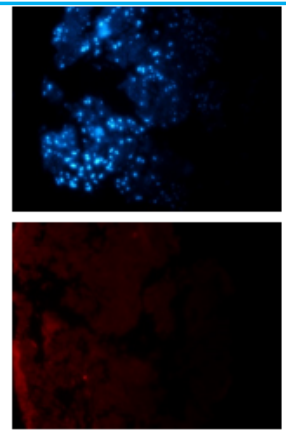

B
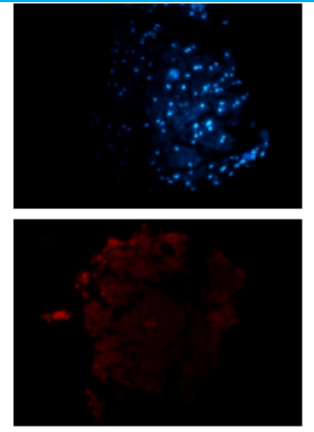

$\mathrm{C}$

FIGURE 1. Immunocytochemistry staining of collagens type 2 and 10 in treated cells; A) collagen type 2 in treatment Dif.a; B) treatment Dif.W and c) collagen 10 in treatment PRP.a (the upper part is staining core by DAPI and the lower part is collagen staining); blue color shows cell core and red color refers to surface place of collagens; the line illustrates size of $0.26 \mathrm{~mm}$ and magnification of image $\times 4$.

alkaline was observed in treatment base.w at 0.32 unit absorption per mg protein. According to obtained results, treatments PRO.W and PRP.a motivate differentiation towards bones more than others and this can be inferred based on activity of alkaline phosphatase in these treatments. Despite to them, treatment base.w has caused lowest stimulation towards boning.

VEGF factor secretion : According to diagram 2, highest secretion level of VEGF was observed in treatments with base environment with PRP. In the base treatment with PRP.a, higher level of Endothelial Angiogenesis Growth Factor was observed compared to base environment in combination with PRP.w. The lowest secretion level was detected in base.w and it seems that the effect of electromagnetic wave treatments and TGF factor varies due to medium culture used. For example, in environment with Dif base, secretion in wave treatment is higher than others; although the results differ in base environment and secretion has been in higher level using TGF treatment. The effects of PRP have been also conflicting. This factor has shown different effects in combination with different culture media exposed to wave and TGF treatments. However, it seems that it has led to increased secretion of VEGF by itself, since secretion in treatments with PRP has been higher than others.

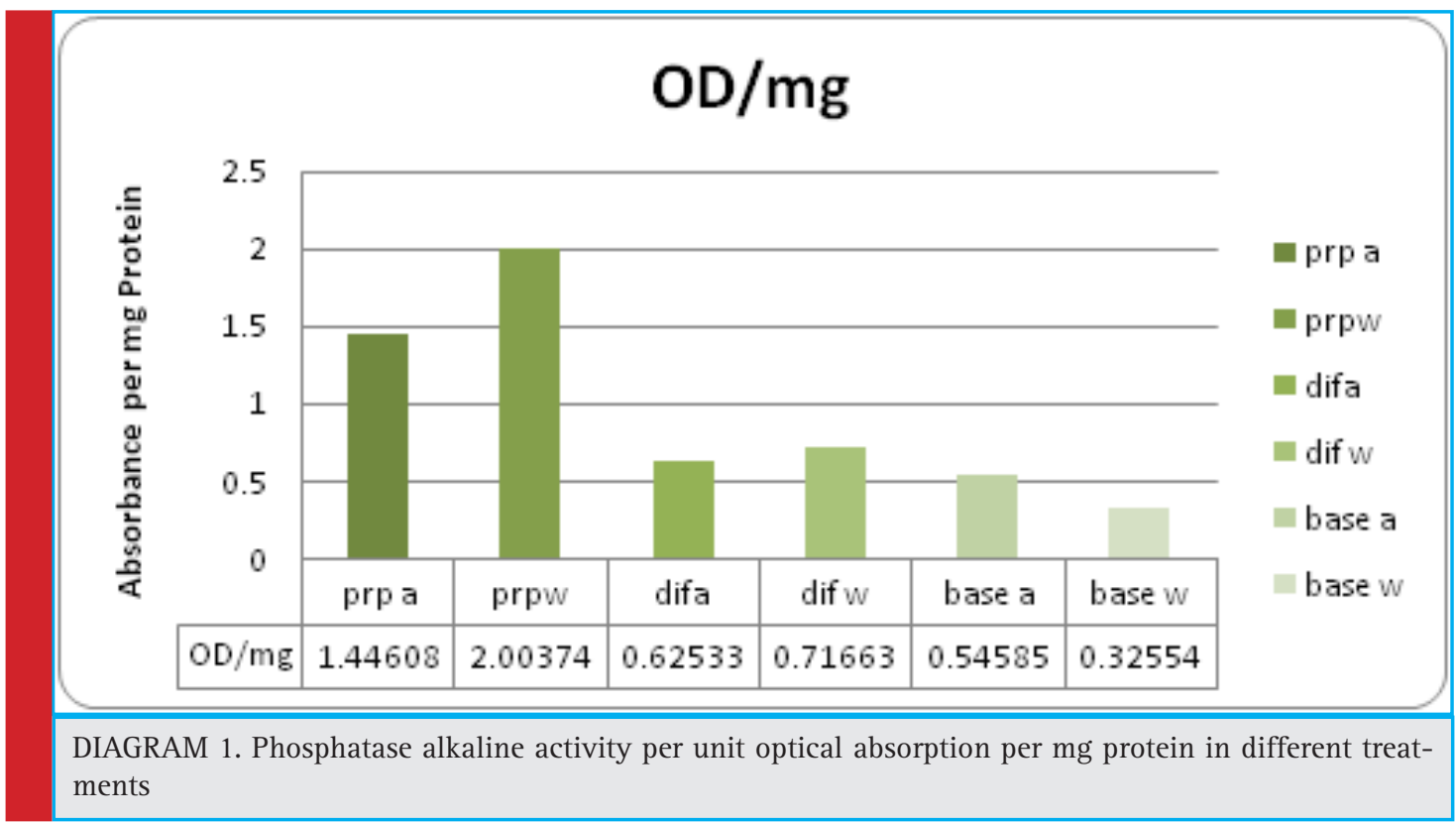

580 


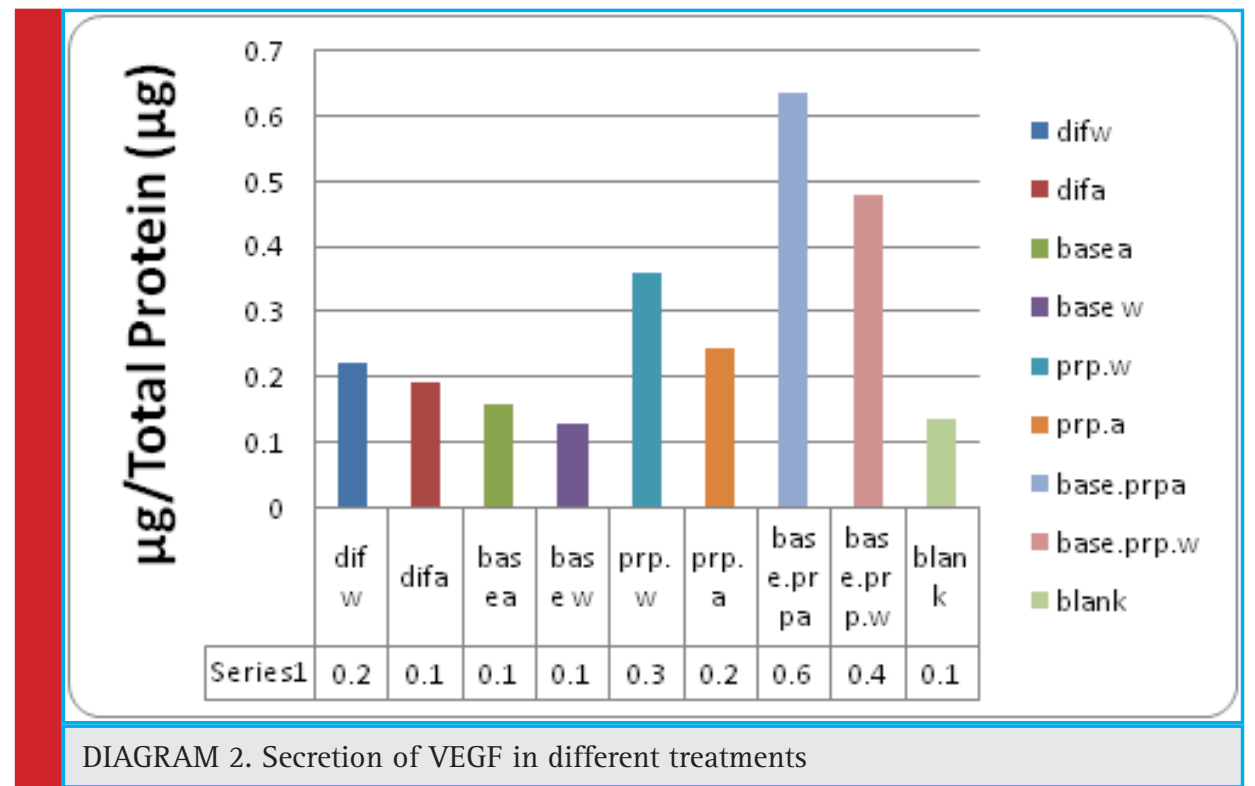

Analysis of inflammation with TGF $\alpha$ measurement: Another symptom of cartilage-related articular diseases is inflammation. According to existing reports, PRP can cause inflammation in some cases and may intensify that. Moreover, it can inhibit and reduce inflammatory reactions. The conflict is because of wide range of inflammatory factors in PRP. Inflammatory potential of engineered tissue has been examined by measurement of TNF $\alpha$. The results have been presented in diagram 3. According to these results, treatments Dif.w and $\mathrm{PRPb}$.a have shown highest level of inflammatory activity. On the contrary, treatments Base.w and Base.A and
PRP.W have shown low level of TNF $\alpha$ even lower than ELISA control level. Treatment Dif showed highest level of inflammation; although same factor showed lowest level of inflammatory activity in combination with Base medium. PRP.b factor has also shown different behavior in different combinations of treatment with TGF and electromagnetic radiation, so that it has shown high inflammatory activity in combination with TGF and inflammatory activity lower than control level in combination with electromagnetic radiation. The conflicting responses can be attributed to complexity of compounds in culture media and PRP compounds, since controlling

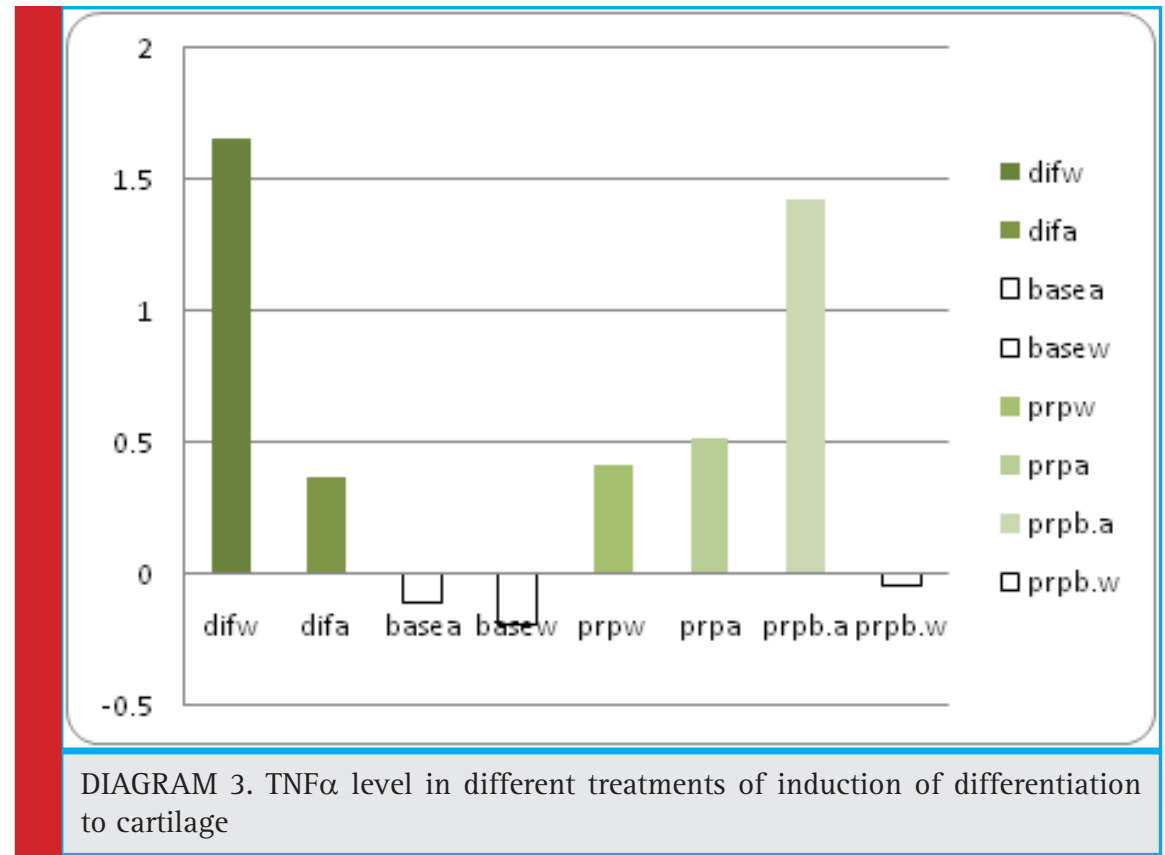




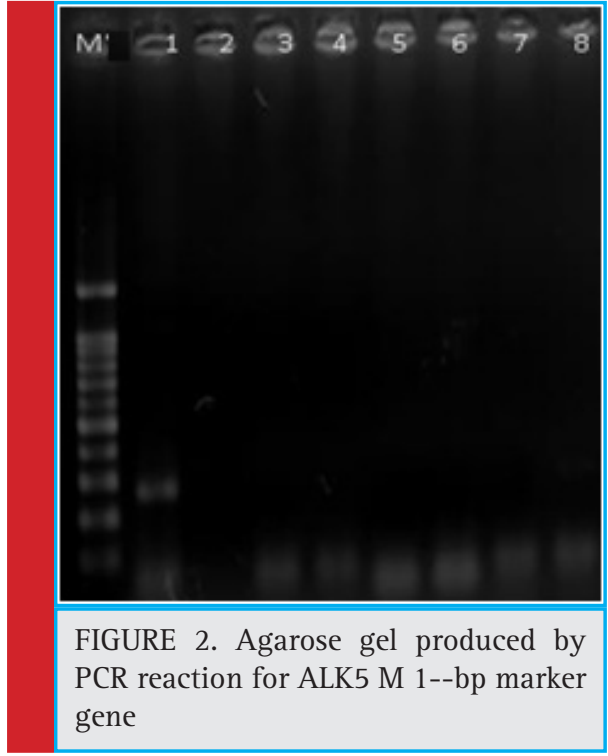

these complex compounds and inhibition of interfering effects of factors in the combinations is difficult.

Measurement of cartilage and bone gene expression : Analysis of cartilage differentiation by gene expression using RT-PCR

Expression of Pleotrophin and ALK5 genes as markers in cartilage differentiation samples and collagen status type 2 gene were analyzed. Collagen type 2 includes 2 isoforms called ColllA and ColllB created as a result of alternative array of exon 2. Expression of these markers during Chondrogenic differentiation caused differentiation efficiency detection. The image of gels produced by reaction of these genes is presented in figures 2 and 3.

Emergence of 250bp band showed expression of ALK5 gene in samples, which can show cartilage differentiation in these samples. 111bp band emergence also

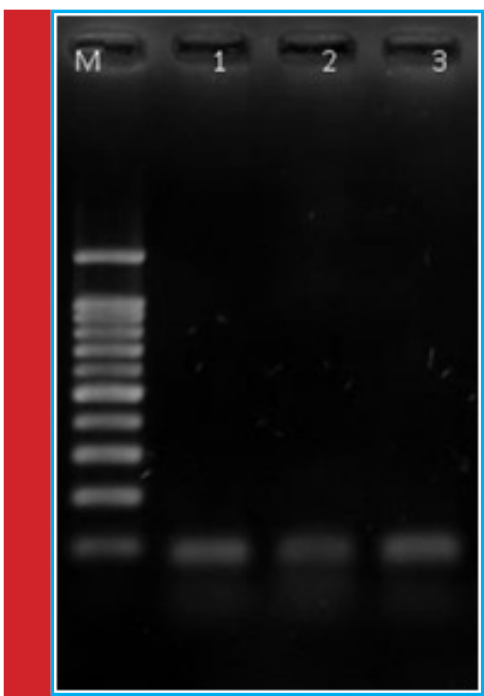

FIGURE 3. Agarose gel produced by PCR reaction for Pleotrophin $\mathrm{M}$ gene $100 \mathrm{bp}$ marker

showed expression of Pleotrophin in cells and confirms cartilage formation processes. However, no band was observed in proliferation of isoforms in collagen type 2 and the result of this gene proliferation was negative, which shows lack expression of this gene in this differentiation steps.

\section{Analysis of specific cartilage genes expression using Real} Time PCR

In order to analyze expression of specific cartilage genes such as sox9, colll and colX using b2m reference gene, Real Time PCR method was used.

Sox9 cartilage marker: Sox9 marker is the transcription factor required for expression of cartilage matrix genes

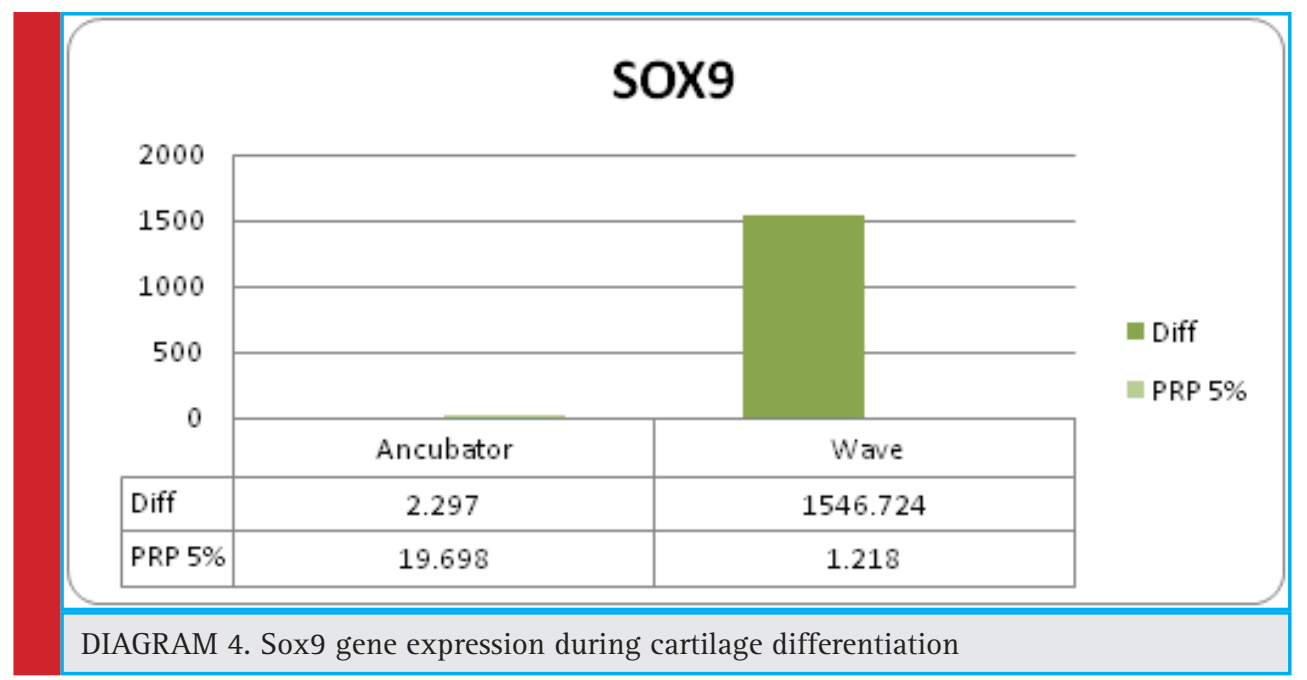




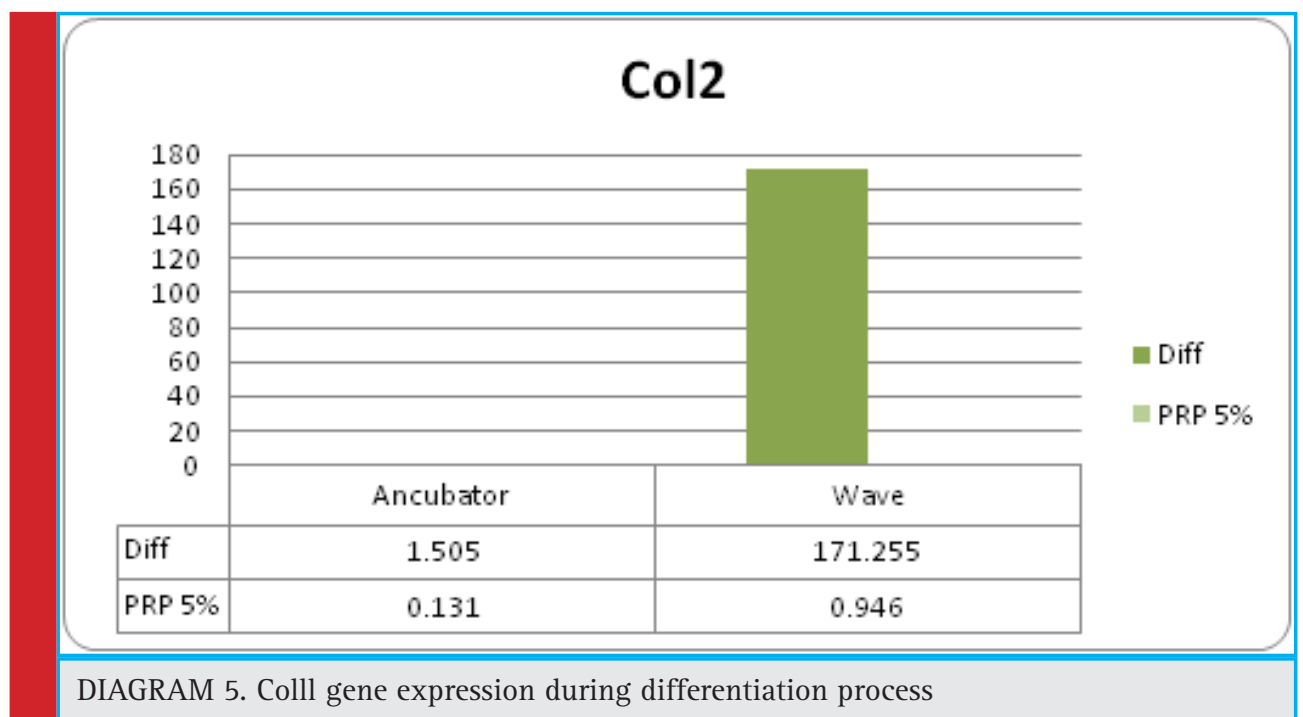

such as collagen type 2. Diagram 4 illustrates sox9 gene expression during cartilage differentiation process. In the day 14 of treatment, expression of this gene in Dif medium exposed to electromagnetic waves was significantly high; although its expression in medium containing 5\% PRP was not tracked. Moreover, expression of this gene was not observed or was insignificant in treatments without wave.

Collagen type 2: The products of this gene were observed in hyaline cartilage in form of fibril. The proteins can't form fiber. In this study, proalpha- 1 chain has been detected, which has interfered in partial production of collagen type 2. According to diagram 5, colll gene expression has been significantly increased under effect of electromagnetic waves. Moreover, the results show that medium containing 5\% PRP has been unable to induce expression of this gene and has also had inhibi- tory effects on wave induction capacity on the other hand.

Collagen type 10: Alpha-1 collagen chain was coded by COL10A1 gene. This gene can encode collagen type-10 alpha chain, which is expressed by hypertrophic chondrocytes during Endocardal bone formation.

Diagram 6 has illustrated adjustment of existing data of electromagnetic wave and 55 PRP on both inhibitory effects on expression of this gene. When the two factors are applied in same treatment on cells, no expression can be observed; although colX gene expression is in high level in presence of the two factors.

\section{DISCUSSION}

Mature stem cells can be used for tissue engineering purposes due to high differentiation ability to several

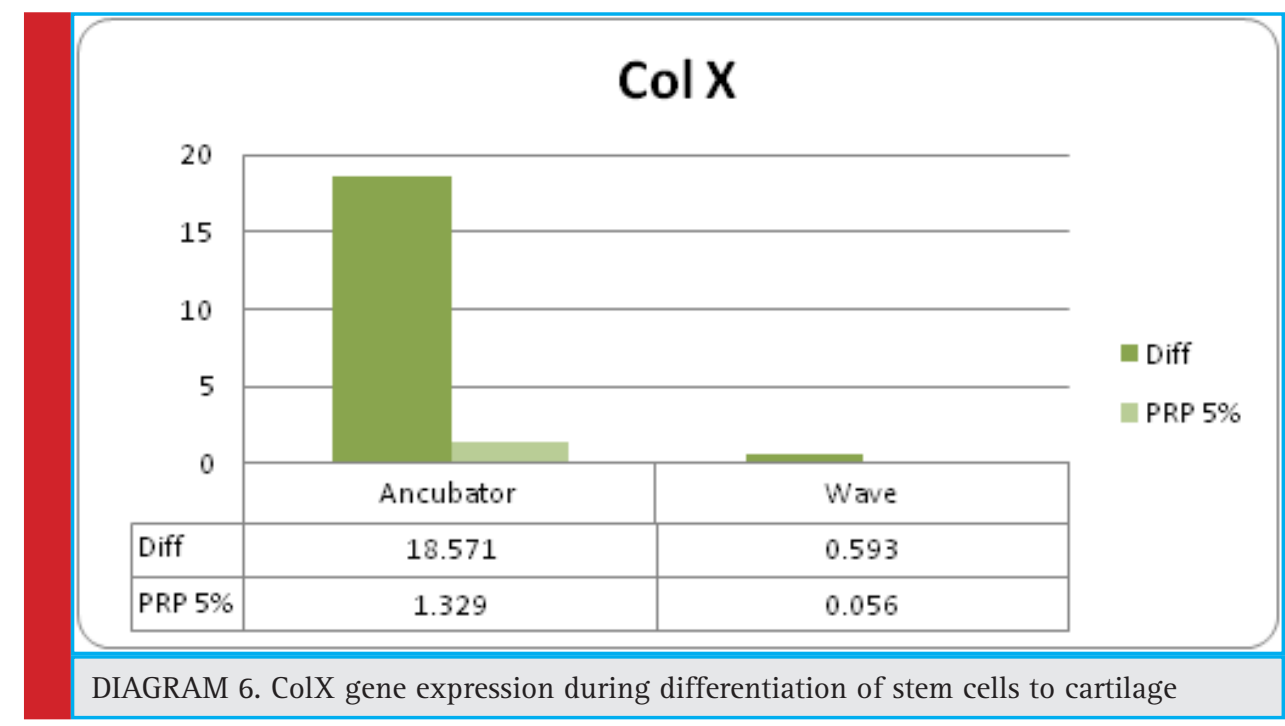


cell types. Recently, fat tissue stem cells have been used to repair tissues. The study conducted by Zul et al. (2001) using stem cell extraction methods based on using collagenase could extract these cells from fat tissue of adult people, (Hunziker et al. 2002). Moreover, Estes Research Team used this method to extract stem cells to obtain fat tissue stem cells to create cartilage phenotype, (Jonstone et al. 1998).

According to findings of Pena et al. (2011), spindle cells with ability of adherence to bottom of culture dish and the ability of differentiation to mesodermic cell lines can be considered as mesenchymal stem cells (Yoo et al. 1998). Adherence ability of cells to plastic surfaces has been used for early separation of stem cell populations. Similar results were also observed in study of Tappe et al. (2009) and Aust et al. (2004), in which nature of fat tissue was analyzed using this type of staining, (Solchaga et al. 2006, Diduch et al. 2000).

In this study, to analyze the differentiation of stem cells extracted from fat tissue to cartilage, expression and production of collagens type 2 and 10 has been applied using Immunocytochemical method. Similar results were also obtained by Ogawa, (Williams et al. 2003). In this study, expression of collagen type 2 was observed during cartilage differentiation and type 10 as bone and hypertrophy markers in chondrocytes. It seems that Dif medium and electromagnetic wave treatment have induced cartilage differentiation and bone markers have been observed in PRP-contained treatment and it seems that the treatment has the ability of inducing hypertrophy and boning. Mishra et al. (2009) showed the effect of PRP on cartilage differentiation. Further, conflicting effects of PRP on cartilage and bone differentiation were revealed (Bosnakovski et al. 2006, Wakitani et al. 2002).

Alkaline phosphatase enzyme activity can be considered as one of the most underlying factors of hypertrophy and bone formation and when the cartilage cells are differentiated, they gain hypertrophic mode and start production of alkaline phosphatase enzyme, (Bruder et al. 1997). According to the results of this study, treatments PRP.W and PRP.a have stimulated differentiation to bone more than others and lowest alkaline phosphatase activity has been observed in treatment Base. $\mathrm{W}$ on the other hand. Measurements showed that highest secretion of VEGF was observed in treatments with base medium containing PRP. Lowest secretion level was also detected in Base. W medium. It is expected that PRP can induce such reaction because of nature rich of growth factors. However, there are some reports on inhibitory effect depended on PRP dose in VEGF secretion (Clin Pediatr Endocrinol 2014). The conflicts can be because of difference in type of treatments, PRP preparation method and its source and also type of cells under treatment.
According to results obtained from this study, treatments Dif.W and PRP.a have shown highest level of inflammatory activity. On the contrary, treatments Base.W, Base.A and PRPb.W have even shown TNF $\alpha$ level even lower than ELISA control level. It seems that combination of base medium lays key role in reduction of inflammatory activities regardless of other inductive factors.

The effects of electromagnetic radiation or using PRP showed different results. For example, radiation in combination with Dif medium showed highest inflammation level; although same factor showed lowest inflammation activity in combination with base medium. In 3 out of 4 treatments containing electromagnetic wave, TNF $\alpha$ level was low and insignificant and it could be inferred that electromagnetic wave can probably reduce inflammatory activity regardless of culture medium compounds.

Controlling expression of cartilage matrix gene like collagen type 2 was taken by transcription factor called sox9. During cartilage differentiation process, the transcription factor played key role and its expression was increased in chondrocytes and Chondroprogenitor cells. According to obtained results, expression of this gene in Dif medium under electromagnetic radiation was significantly increased; although its expression was not detected in medium containing 5\% PRP. Moreover, the expression of this gene was not observed or was insignificant in treatments without wave. As a result of increased expression of sox 9 gene, its downstream gene (colll) was also increased in terms of expression. Moreover, the results showed that medium containing 5\% PRP has not the ability to induce expression of this gene and has had also inhibitory effects on wave induction capacity on the other hand. Colll expression pattern under treatment has been significantly similar to expression pattern of control gene (sox9).

Adjustment of data of electromagnetic wave and 5\% PRP has shown that both of them have inhibitory effects on colX gene expression.

\section{CONCLUSION}

The results of the present study showed the efficient and logical use of PRP and electromagnetic waves in medicine and tissue engineering. However PRP allow cartilage differentiation in the right format, this method need further study and cannot decrease pathologic symptoms.

\section{ACKNOWLEDGMENT}

This research was an independent bachelors project supported by research affairs University of Tehran, Tehran, Iran with cooperation of Mrs. Mahsa Laleh at Azad University of Science and Research Branch, Tehran, Iran. 


\section{REFERENCES}

Agnes S. Klar, Jakub Zimoch, and Thomas Biedermann, Skin Tissue Engineering: Application of Adipose-Derived Stem Cells, BioMed Research International, vol. 2017, Article ID 9747010, 12 pages, 2017. doi:10.1155/2017/9747010

Alicja Zajdel, Magdalena Kałucka, Edyta Kokoszka-Mikołaj, Adam Wilczok (2017). Osteogenic differentiation of human mesenchymal stem cells from adipose tissue and Wharton's jelly of the umbilical cord. Acta Biochimica Polonica. 2017 no. 64 Issue 2 pp 365-369

Anitua, E., et al., (2007) The potential impact of the preparation rich in growth factors (PRGF) in different medical fields. Biomaterials, 2007. 28(31): p. 4551-60.

Beata Kocan, Aleksandra Maziarz, Jacek Tabarkiewicz, (2017) Trophic Activity and Phenotype of Adipose Tissue-Derived Mesenchymal Stem Cells as a Background of Their Regenerative Potential, Stem Cells International, Article ID 1653254, 13 pages doi:10.1155/2017/1653254

Bosnakovski, D., et al., (2006) Chondrogenic differentiation of bovine bone marrow mesenchymal stem cells (MSCs) in different hydrogels: influence of collagen type II extracellular matrix on MSC chondrogenesis. Biotechnology and Bioengineering, 93(6): p. 1152-1163.

Bruder, S.P., N. Jaiswal, and S.E. Haynesworth (1997), Growth kinetics, self-renewal, and the osteogenic potential of purified human mesenchymal stem cells during extensive subcultivation and following cryopreservation. Journal of cellular biochemistry, 1997. 64(2): p. 278-294.

Choi JR, Yong KW, Choi JY (2018). Effects of mechanical loading on human mesenchymal stem cells for cartilage tissue engineering. J Cell Physiol. 233(3):1913-1928. doi: 10.1002/ jcp.26018. Epub 2017 Jun 12. DOI: 10.1002/jcp.26018

Diduch, D.R., et al., (2000) Marrow stromal cells embedded in alginate for repair of osteochondral defects. Arthroscopy: The Journal of Arthroscopic \& Related Surgery, 16(6): p. 571-577.

Elder, S. and J. Thomason (2014) Effect of Platelet-Rich Plasma on Chondrogenic Differentiation in Three-Dimensional Culture. The Open Orthopaedics Journal. 8: p. 78-84.

Fukaya, M. and A. Ito, (2014) A New Economic Method for Preparing Platelet-rich Plasma. Plastic and Reconstructive Surgery Global Open, 2(6): p. e162.

Gugjoo, M. B., Amarpal, Sharma, G. T., Aithal, H. P., \&t Kinjavdekar, P. (2016). Cartilage tissue engineering: Role of mesenchymal stem cells along with growth factors \&t scaffolds. The Indian Journal of Medical Research, 144 (3), 339-347. http:// doi.org/10.4103/0971-5916.198724

Heo JS, Choi Y, Kim HS, Kim HO (2015). Comparison of molecular profiles of human mesenchymal stem cells derived from bone marrow, umbilical cord blood, placenta and adipose tissue. Int J Mol Med. 2016 Jan;37(1):115-25. doi: 10.3892/ ijmm.2015.2413. Epub 2015 Nov 19.

Hunziker, E., Articular cartilage repair: basic science and clinical progress. A review of the current status and prospects. Osteoarthritis and cartilage, 2002. 10(6): p. 432-463.
Johnstone, B., et al. (1998) In Vitro Chondrogenesis of Bone Marrow-Derived Mesenchymal Progenitor Cells. Experimental cell research, 238(1): p. 265-272.

Judit Iván, Evelin Major, Adrienn (2017). The Short-Chain Fatty Acid Propionate Inhibits Adipogenic Differentiation of Human Chorion-Derived Mesenchymal Stem Cells Through the Free Fatty Acid Receptor 2. Stem Cells Dev. December 1; 26(23): 1724-1733.Doi: 10.1089/scd.2017.0035

Lee, H.R., et al., (2012) Platelet-rich plasma loaded hydrogel scaffold enhances chondrogenic differentiation and maturation with up-regulation of CB1 and CB2. J Control Release, 159(3): p. 332-7.

Lei Tan, Bin Zhao, Fu-Tao Ge, Da-Hui Sun \& Tiecheng Yu (2017). Shockwaves Inhibit Chondrogenic Differentiation of Human Mesenchymal Stem Cells in Association with Adenosine and A2B Receptors. Scientific Reports 7, Article number: 14377 (2017). doi:10.1038/s41598-017-14875-y

Liu Z, Yuan X, Fernandes G, Dziak R, Ionita CN, Li C, Wang C, Yang S (2017). The combination of nano-calcium sulfate/ platelet rich plasma gel scaffold with BMP2 gene-modified mesenchymal stem cells promotes bone regeneration in rat critical-sized calvarial defects. Stem Cell Res Ther. 2017 May 25;8(1):122. doi: 10.1186/s13287-017-0574-6. DOI: 10.1186/ s13287-017-0574-6

Liu, X., Ren, W., Jiang, Z., Su, Z., Ma, X., Li, Y.,Yang, X. (2017). Hypothermia inhibits the proliferation of bone marrowderived mesenchymal stem cells and increases tolerance to hypoxia by enhancing SUM Oylation. International Journal of Molecular Medicine, 40(6), 1631-1638. http://doi.org/10.3892/ ijmm.2017.3167

Maryna Bondarava, Chiara Cattaneo, Bin Ren, Wolfgang E. Thasler, Volkmar Jansson, Peter E. Müller \&t Oliver B. Betz (2017). Osseous differentiation of human fat tissue grafts: From tissue engineering to tissue differentiation. Scientific Reports volume 7, Article number: 39712 (2017). doi:10.1038/srep39712

Mobini, S., Leppik, L., Thottakkattumana Parameswaran, V., \& Barker, J. H. (2017). In vitro effect of direct current electrical stimulation on rat mesenchymal stem cells. PeerJ, 5, e2821. http://doi.org/10.7717/peerj.2821

Petrera, M., et al., (2011) Supplementation With Platelet-Rich Plasma Improves the InVitro Formation of Tissue-Engineered Cartilage With Enhanced Mechanical Properties. Arthroscopy. 29(10): p. 1685-1692.

Qian, Y., Han, Q., Chen, W., Song, J., Zhao, X., Ouyang, Y. Fan, C. (2017). Platelet-Rich Plasma Derived Growth Factors Contribute to Stem Cell Differentiation in Musculoskeletal Regeneration. Frontiers in Chemistry, 5, 89. http://doi.org/10.3389/ fchem.2017.00089

Ross, C. L., Siriwardane, M., Almeida-Porada, G., Porada, C. D., Brink, P., Christ, G. J., \& Harrison, B. S. (2015). The effect of low-frequency electromagnetic field on human bone marrow stem/progenitor cell differentiation. Stem Cell Research, 15(1), 96-108. http://doi.org/10.1016/j.scr.2015.04.009

Rubio-Azpeitia, E. and I. Andia, (2014) Partnership between platelet-rich plasma and mesenchymal stem cells: in vitro 
experience. Muscles, Ligaments and Tendons Journal 4(1): p. 52-62.

S.G. Dubois et al. (2008) Isolation of Human Adipose-derived Stem Cells from Biopsies and Liposuction Specimens 208

Seong Y, Moon J, Kim J. Life Sci. 2014 Apr 25; 102(1):16-27. Epub 2014 Mar 3.

Solchaga, L.A., et al., (2006) A rapid seeding technique for the assembly of large cell/scaffold composite constructs. Tissue Engineering 12(7): p. 1851-1863.

Tan, A. R., \&t Hung, C. T. (2017). Mesenchymal Stem Cells for Functional Cartilage Tissue Engineering: Taking Cues from Chondrocyte-Based Constructs. Stem Cells Translational Medicine, 6(4), 1295-1303. http://doi.org/10.1002/sctm.16-0271

Toshimi Michigami (2014) Current Understanding on the Molecular Basis of Chondrogenesis Clin Pediatr Endocrinol. 2014 Jan; 23(1): p,1-8.

Vivian Alonso-Goulart, Lorraine Braga, Ferreira, (2017). Mesenchymal stem cells from human adipose tissue and bone repair: a literature review. Biotechnology Research and Innovation. Available online 14 November 2017. https://doi. org/10.1016/j.biori.2017.10.005
Wakitani, HJ et al. (2002) Human autologous culture expanded bone marrow mesenchymal cell transplantation for repair of cartilage defects in osteoarthritic knees. Osteoarthritis and cartilage 2002. 10(3): p. 199-206.

Williams, C.G., et al., (2003) In vitro chondrogenesis of bone marrow-derived mesenchymal stem cells in a photopolymerizing hydrogel. Tissue Engineering, 2003. 9(4): p. 679-688.

Xu Q, Li B, Yuan L, Dong Z, Zhang H, Wang H, Sun J, Ge S, Jin Y (2017). Combination of platelet-rich plasma within periodontal ligament stem cell sheets enhances cell differentiation and matrix production. J Tissue Eng Regen Med. 11(3):627636. doi: 10.1002/term.1953. Epub 2014 Sep 4. DOI: 10.1002/ term. 1953

Yoo, J.U., et al., (1998) The Chondrogenic Potential of Human Bone-Marrow-Derived Mesenchymal Progenitor Cells The Journal of Bone \& Joint Surgery 80(12): p. 1745-

Zhai L, Ma XL, Jiang C, Zhang B, Liu ST, Xing GY (2016) Human autologous mesenchymal stem cells with extracorporeal shock wave therapy for nonunion of long bones. Indian J Orthop [serial online] 2016 [cited 2018 Jan 28;50:543-50. Available from: http://www.ijoonline.com/text. asp?2016/50/5/543/189602 